\title{
INFLUÊNCIA DO PH NA SORÇÃO DE IMAZAQUIN EM UM LATOSSOLO VERMELHO ACRIFÉRRICO(1)
}

\author{
W. S. D. ROCHA ${ }^{(2)}$, L.R. F. ALLEONI(3), J . B. REGITANO(4), \\ J. C. CASAGRANDE ${ }^{(5)} \&$ V. L. TORNISIELLO(6)
}

\begin{abstract}
RESUMO
O herbicida imazaquin é uma molécula orgânica com grupos funcionais ionizáveis, sendo sua sorção dependente do pH e da carga elétrica líquida do solo. Neste trabalho, foi estudada a sorção do imazaquin nas camadas superficiais e subsuperficiais de um Latossolo Vermelho Acriférrico típico textura muito argilosa, considerando quatro diferentes valores de pH. Com uso de moléculas radiomarcadas com carbono-14, determinou-se a quantidade sorvida pela diferença entre a quantidade aplicada e a remanescenteem solução. Os resultados foram ajustados à equação de $F$ reundlich, para determi nação do coeficiente de sorção $\left(K_{f}\right)$. De maneira geral, o herbicida apresentou baixa sorção no solo. Nas amostras coletadas nas duas profundidades, a sorção diminuiu com a elevação do pH. Para um mesmo valor de pH, a sorção foi maior no horizonte subsuperficial do que no superficial, sendo a carga líquida positiva resultante do baixo teor de matéria orgânica e dos elevados conteúdos de óxidos de ferro e de alumínio. Quando a carga elétrica líquida do solo foi positiva, não foi possível predizer a sorção de imazaquin, considerando apenas a especiação da molécula e a sua partição na fração orgânica do solo.
\end{abstract}

Termos de indexação: her bicida, imazaquim, pH, sorção, ácrico.

(1) Parte da Tese de Mestrado do primeiro autor, apresentada à Escola Superior de Agricultura "Luiz de Queiroz" - ESALQ, Piracicaba (SP). Recebido para publicação em novembro de 1999 e aprovado em maio de 2000.

(2) Engenheiro-Agrônomo, Doutorando em Solos e Nutrição de Plantas - ESALQ/USP, Caixa Postal 9, CEP 13418-900 Piracicaba (SP). Bolsista do CNPq. E-mail: wsdrocha@carpa.ciagri.usp.br

(3) Professor do Departamento de Solos e Nutrição de Plantas - ESALQ/USP. Bolsista do CNPq. E-mail: Irfalleo@carpa.ciagri.usp.br (4) Pesquisadora da Seção de E cotoxicol ogia do Centro de Energia Nuclear na Agricultura - CE NA/USP, Caixa Postal 96, CEP 13400970 Piracicaba (SP). E-mail: regitano@cena.usp.br

(5) Professor do Centro de Ciências Agrárias da Universidade Federal de São Carlos - UFSCar, Caixa Postal 153, CEP 13600-970 Araras (SP). Bolsista do CNPq. E-mail: bighouse@power.ufscar.br

(6) Pesquisador da Seção de E cotoxicologia do Centro de Energia Nuclear na Agricultura - CE NA/USP. E-mail: vitornis@cena.usp.br 


\title{
SUMMARY: INFLUENCE OF PH IN IMAZAQUIN SORPTION ON AN ANIONIC ACRUDOX
}

\begin{abstract}
I mazaquin herbicide is an organic molecule presenting ionizable functional groups, with its sorption depending on soil pH and electrical charges. In this research, sorption of imazaquin was evaluated on an Anionic Acrudox at different soil depths and pH values. Radiolabelled $\left({ }^{14} \mathrm{C}\right)$ molecules were employed to study imazaquin sorption, which was estimated by the difference between applied concentration and that remaining in solution. The sorption data were fitted to F reundlich equation to determine the sorption coefficient values $\left(\mathrm{K}_{\mathrm{f}}\right)$, which were, overall, low in thestudied soil . At both soil depths, sorption decreased as $\mathrm{pH}$ increased. However, at a similar $\mathrm{pH}$ value, sorption was higher on the subsurface than on the surface sample, probably due to the positive charges resulting from the low organic matter and high Al and Feoxidecontents. When thesoil surfacecharges werepositive it was not possible to predict imazaquin sorption only from mol ecule speciation and its hydrophobic partition into soil organic fractions.
\end{abstract}

Index terms: herbicide, imazaquin, $\mathrm{pH}$, sorption, acric.

\section{INTRODUÇÃO}

A contaminação de solos e águas pelo uso de pesticidas pode comprometer os ecossistemas ambientais ea saúde dohomem. Para prevenir estes problemas, é preciso conhecer a dinâmica dos pesticidas no ambiente após sua aplicação, que é bastante influenciada pelo mecanismo de sorção (retenção) às partículas do solo. Para compreender os mecanismos de sorção de um pesticida ao solo, é fundamental conhecer a composição da matriz do solo (Koskinen \& Harper, 1990), além das características físico-químicas da molécula.

I mazaquin (ácido 2-[4,5-dihidro-4-metil-4-(1metiletil)-5-oxo-1H-imidazol-2-il]-3-quinol inacarboxílico) é um herbicida de uso intensivo no Brasil, com amplo espectro de controle de plantas daninhas associadas à cultura da soja (Goetz et al., 1986; Basham et al., 1987). A molécula contém dois grupos funcionais ionizáveis: um grupo carboxílico (ácido, $\mathrm{pK}_{\mathrm{a}}=3,8$ ) e um grupo quinolina (básico, $\mathrm{pK}_{\mathrm{a}}=2,0$ ) (Stougaard et al., 1990). Dada sua natureza anfótera, fatores relacionados com o solo, comoteor de carbono orgânico (C.O.), pH , força iônica e potencial elétrico, devem ser considerados para estimar a sorção do imazaquin. Para solos com carga permanente negativa, tem-seobservado que a sorção de imazaquin é diretamente proporcional ao teor de c.O. (correspondente ao número de sítios hidrofóbicos) einversamente proporcional ao pH do solo (especiação da molécula).

Regitano et al. (1997) propuseram o seguinte modelo para quantificar a sorção de imazaquin nestes solos: $\mathrm{K}_{\mathrm{oc}}=\mathrm{K}_{\mathrm{oc}, \mathrm{n}} \phi_{\mathrm{n}}+\mathrm{K}_{\mathrm{oc}, \mathrm{a}}\left(1-\phi_{\mathrm{n}}\right)$, sendo resultante da soma dos coeficientes de sorção normalizada em relação aos teores deC.O. $\left(K_{o c}\right)$ para as espécies neutras $\left(K_{o c, n}\right)$ eaniônicas $\left(K_{o c, a}\right)$, na qual $\phi_{\mathrm{n}}$ representa a proporção de espécies neutras. Os autores verificaram que alguns solos com maior quantidade de óxidos, hidróxidos e C.O. apresentaram desvios em relação ao modelo, demonstrando que a sorção do imazaquin estava sendo influenciada por interações não-hidrofóbicas com a matéria orgânica ou superfícies minerais.

$\mathrm{Na}$ faixa de $\mathrm{pH}$ mais comum dos solos tropicais agricultáveis $(5,0$ a 6,5$)$, o imazaquin comporta-se predominantemente como ânion orgânico. Como a maioria dos sol os tem carga el étrica líquida negativa, ocorre baixa sorção do imazaquin aos seus colóides em virtude das forças eletrostáticas repulsivas (Renner et al., 1988; Pires et al., 1997). Com decréscimo do $\mathrm{pH}$ do solo, o imazaquin converte-se para a forma neutra e, nessa condição, as interações hidrofóbicas com a matéria orgânica do sol oacentuamse (Best \& Weber, 1974; Col bert et al., 1975).

A maioria dos trabalhos relativos à sorção de pesticidas citados na literatura refere-se à camada superficial (0-0,2 m) de solos de regiões com clima temperado, os quais normal menteapresentam altos teores de matéria orgânica e carga elétrica negativa permanentena superfície de seus colóides, ou a sol os de regiões tropicais também com carga líquida negativa.

Alguns Latossolos, entretanto, têm comportamento físico-químico diferenciado, pois a carga elétrica de seus colóides é variável, ou seja, eles podem exibir balanço de cargas negativo ou positivo, dependendo das condições do meio. Como exemplo, estão os ácricos, que representam o extremo na escala de intemperismo e podem apresentar carga líquida positiva no horizonte B (Alleoni \& Camargo, 1995), graças aos el evados teores de óxidos de ferro e de alumínio e aos baixos teores de matéria orgânica, gerando valores de ponto de efeito salino nulo 
(PESN) superiores aos de pH em água. Neste horizonte, a retenção de ânions (inorgânicos e orgânicos) pode ser superior à de cátions (Alleoni \& Camargo, 1994), podendo diminuir o risco de contaminação de águas superficiais e, ou, subterrâneas. Os sol os ácricos têm capacidadeefetiva de troca de cátions $(\mathrm{Ca}+\mathrm{Mg}+\mathrm{K}+\mathrm{Na}+\mathrm{Al})$ menor do que $15 \mathrm{mmol}_{\mathrm{C}} \mathrm{kg}^{-1}$ de argila e $\mathrm{pH}$ medido em $\mathrm{KCl}$ $1,0 \mathrm{~mol} \mathrm{~L}^{-1}$ superior ou igual a 5,0 , ou $\Delta \mathrm{pH}\left(\mathrm{pH}_{\mathrm{KCl}}-\right.$ $\mathrm{pH}_{\mathrm{H}_{2} \mathrm{O}}$ ) positivo ou nulo (EMBRAPA, 1999).

Quanto maior o nível de detalhamento dos levantamentos, maior a probabilidade de se encontrarem solos classificados como ácricos. Conseqüentemente, torna-se necessário conhecer mel hor o comportamento de corretivos, fertilizantes e defensivos agrícolas nesse tipo de solo. Neste trabalho, estudou-se o efeito do pH do sol o na sorção do herbicida imazaquin em um L atossolo Vermel ho Acriférrico típico textura muito argil osa, coletado em superfície e em profundidade. Avaliou-se, também, o modelo de Regitano et al. (1997) quanto à sua capacidade de estimar a sorção do referido herbicida nesse tipo de solo.

\section{MATE RIAL E MÉTODOS}

Foram utilizadas amostras de um Latossolo Vermelho Acriférrico típico textura muito argilosa, local izado no município de Ribeirão Preto, coletadas na camada superficial $(0$ a $0,2 \mathrm{~m})$ e na de maior expressão do horizonte B (1,0 a 1,4 m).

Os métodos para as análises químicas, físicas e mineralógicas, real izadas no Departamento de Sol os e Nutrição de Plantas da Escola Superior de Agricultura "Luiz de Queiroz", encontram-se em Camargo et al. (1986). O C.O. foi obtido após oxidação da matéria orgânica do solo com solução de dicromato de potássio em presença deáci do sulfúrico e titulação do excesso de dicromato com sulfato ferroso amoniacal. Os teores de óxidos $\left(\mathrm{SiO}_{2}, \mathrm{Fe}_{2} \mathrm{O}_{3}\right.$ e $\mathrm{Al}_{2} \mathrm{O}_{3}$ ) foram obtidos após ataque com ácido sulfúrico, e com el es foram cal culados os índices de intemperismo: $\mathrm{Ki}=\left(\mathrm{SiO}_{2} / \mathrm{Al}_{2} \mathrm{O}_{3}\right) \times 1,7 \mathrm{e} \mathrm{Kr}=\left\{\left(\mathrm{SiO}_{2} /\right.\right.$ $\left.60) /\left[\left(\mathrm{Al}_{2} \mathrm{O}_{3} / 102\right)+\left(\mathrm{Fe}_{2} \mathrm{O}_{3} / 160\right)\right]\right\}$. A quantidade de ferro $\left(\mathrm{Fe}_{\mathrm{d}}\right)$ ealumínio $\left(\mathrm{Al}_{\mathrm{d}}\right)$ foi extraída com ditionitocitrato-bicarbonato de sódio $(\mathrm{pH} 7,3)$, tendo sido o ferro reduzido pelo ditionito de sódio e complexado pelo citrato de sódio, permanecendo em solução. A análise granulométrica foi feita pelo método do densímetro. A gibbsita e a caulinita foram quantificadas por meio da Análise Térmica Diferencial (ATD) na fração argila deferrificada e isenta de matéria orgânica.

Com base no método descrito por Barrow (1983, 1984), as amostras de solo (4 kg) foram incubadas com $\mathrm{CaCO}_{3}$ p.a. e $\mathrm{HCl} 1 \mathrm{~mol} \mathrm{~L}^{-1}$ para obter quatro valores de $\mathrm{pH}$ na faixa de 3,0 a 8,0. As amostras de superfície foram incubadas com 3 doses de $\mathrm{CaCO}_{3}$ $(4,8$ e $32 \mathrm{~g})$ e 1 dose de $\mathrm{HCl}(320 \mathrm{~mL})$, e as amostras de profundidade foram incubadas com 2 doses de $\mathrm{CaCO}_{3}(4$ e $8 \mathrm{~g}$ ) e 2 doses de $\mathrm{HCl}(160$ e $320 \mathrm{~mL}$ ), além das testemunhas (sem $\mathrm{CaCO}_{3}$ e sem $\mathrm{HCl}$ ). A umi dade do sol o foi mantida constante durantetoda incubação (30\% em relação à massa de TFSA), até atingir pH constante (atingido após 90 dias). Posteriormente, as amostras foram secas ao ar, peneiradas ( $2 \mathrm{~mm}$ ) e armazenadas em sacos plásticos. Nessas amostras, foi obtido o $\mathrm{pH}$ em $\mathrm{CaCl}_{2}$ $0,01 \mathrm{~mol} \mathrm{~L}^{-1}$. O ponto de efeito salino nulo (PESN) foi obtido pelo método da titulação com $\mathrm{H}^{+}$e $\mathrm{OH}^{-}$, em três diferentes concentrações de $\mathrm{KCl}(0,1 ; 0,01$ e 0,001 mol L-1), correspondendo o PESN ao ponto de intersecção das curvas de titulação. Na seqüência, foi calculado o potencial el étrico superficial $\left(\Psi_{0}\right)$ por meio da equação $\Psi_{0}=59,1$ (PESN - pH).

O estudo de sorção foi feito no centro de Energia Nuclear na Agricultura da USP, em Piracicaba (SP). Para obtenção das isotermas de sorção, foi empregada molécula radiomarcada de imazaquin ${ }^{(14} \mathrm{C}$ ) com atividade específica $=0,80 \mathrm{MBq} \mathrm{mg}^{-1} \mathrm{e}$ pureza $=98 \%$. Alíquotas de $5 \mathrm{~mL}$ de cinco soluções de imazaquin $\left(2,4,8,16\right.$ e $32 \mu \mathrm{mol} L-1$, em $\mathrm{CaCl}_{2}$ 0,01 mol L-1) foram adicionadas a 2,00 g deamostras de terra fina seca ao ar, em triplicata, devidamente acondicionadas em tubos de centrífuga $(50 \mathrm{~mL})$. As amostras foram agitadas em sala climatizada a $25 \pm 2^{\circ} \mathrm{C}$, por $24 \mathrm{~h}$, a 200 agitações min-1. A pós este período, os tubos com as amostras foram centrifugados a 10.000 rpm, por 15 min, ealíquotas de $1 \mathrm{~mL}$ dos sobrenadantes foram retiradas e adicionadas a $10 \mathrm{~mL}$ de solução cintiladora, para determinar a concentração de imazaquin em equilíbrio na solução $\left(C_{e}\right)$, por meio de contagem em cintilador líqui do (CL). A concentração deimazaquin retido foi calculada pela diferença entre a concentração inicial $\left(C_{i}\right)$ ea concentração em solução $\left(\mathrm{C}_{\mathrm{e}}\right)$ após atingir equilíbrio. Posteriormente, os sobrenadantes foram descartados e os tubos pesados para determinar a quantidade de imazaquin remanescente na solução.

Para determinar a percentagem de imazaquin extraída das diferentes amostras de solo, alíquotas de $10 \mathrm{~mL}$ de solução de $\mathrm{CaCl}_{2}$ 0,01 mol L-1 foram adicionadas aos tubos, que foram agitados e centrifugados, conforme real izado no teste de sorção. Posteriormente, alíquotas de $1 \mathrm{~mL}$ dos sobrenadantes foram retiradas e adicionadas a $10 \mathrm{~mL}$ de solução cintiladora para determinar a concentração de imazaquin em solução. O mesmo procedimento foi repetido mais uma vez. Finalizando, as amostras de sol os remanescentes no fundo dos tubos foram secas ao ar, homogeneizadas, pesadas e submetidas à combustão para determinar a quantidade de imazaquin retida no solo.

Os resultados de sorção foram ajustados à equação linearizada de Freundlich $\log S=\log K_{f}+N$ $\log _{e}$, na qual $S=$ quantidade de imazaquin sorvida, 
$\mathrm{K}_{\mathrm{f}}=$ constante de sorção de Freundlich, $\mathrm{C}_{\mathrm{e}}=$ concentração de imazaquin na solução e $\mathrm{N}=$ declividade da isoterma. Para efetuar comparações entre as amostras e o modelo de Regitano et al. (1997), o coeficiente de partição $\left(K_{d}\right)$ foi calculado, assumindo $\mathrm{N}=1$ e normalizado para o teor de C.O. do sol o, obtendo-se, assim, o valor de $\mathrm{K}_{o c}\left(\mathrm{~K}_{\mathrm{oc}}=\mathrm{K}_{\mathrm{d}}\right)$ C.O.).

\section{RESULTADOSE DISCUSSÃO}

Os resultados das análises físicas e químicas do solo, em superfície e em profundidade, encontramse no quadro 1. Trata-se de um solo altamente intemperizado, classificado como gibbsítico sesquioxídico, $\mathrm{Ki} \leq 0,75$ e $\mathrm{Kr}<0,75$, de acordo com Resende \& Santana (1988), com elevados teores de argila e óxidos de alumínio e de ferro. Em média, $50 \%$ do total deóxidos de ferro encontra-se na forma mais cristalina. Sabe-se que o óxido de ferro é o principal responsável pela geração de cargas positivas neste tipo de solo com carga variável (Yu, 1997).

Na camada superficial (0-0,2 m), o val or do ponto de efeito salino nulo (PESN) baixo deveu-se à presença da matéria orgânica (PESN =2, Uehara, 1988) que, provavelmente, estaria revestindo a superfície dos óxidos (PESN entre 7,5 e 9,0, Bell \& Gillman, 1978), bloqueando, assim, os sítios de carga positiva. Em profundidade, o PESN foi mais alto em decorrência do baixo teor de matéria orgânica e do maior conteúdo de gibbsita e óxidos de ferro. O potencial elétrico do solo foi positivo sempre que o pH do sol o mostrou-se menor queoPESN, fenômeno este observado, em profundidade, para os val ores de $\mathrm{pH} 3,5$ e 5,1, e, em superfície, para o pH 3,4 (Quadro 2).

Os val ores dos coeficientes de sorção de F reundlich $\left(K_{f}\right)$ e do coeficientede partição $\left(K_{d}\right)$ estão apresentados no quadro 2. De forma geral, a sorção do imazaquin foi baixa, independentemente da profundidade de amostragem e do valor de $\mathrm{pH}$ do solo. À exceção das amostras subsuperficiais, com valores de $\mathrm{pH}$ 3,5 e 5,1 , e da superficial, com pH 3,4, na qual os valores do potencial elétrico do sol o foram positivos, isso seria esperado, uma vez que mais de $80 \%$ das moléculas de imazaquin apresentaram-se na forma aniônica ( $\phi_{a}$, Quadro 2) e, portanto, foram repelidas pelas cargas negativas do solo.

A figura 1 apresenta os valores da concentração de equilíbrio $\left(\mathrm{C}_{\mathrm{e}}\right)$ versus a quantidade sorvida (S) de imazaquin nos diferentes val ores de $\mathrm{pH}$, ajustados pela equação linearizada de Freundlich. A quantidade de moléculas sorvidas aumentou à medida queaumentou a concentração de imazaquin em solução, constatação esta também feita por Hang et al. (1996). Ao longo do intervalo de concentração estudado, este aumento foi praticamente linear ( $N$ variou de 0,86 a 0,98, Quadro 2). Resultados semel hantes foram obtidos em outras pesquisas (Loux et al., 1989; Regitano et al., 1997).

Quanto maior o valor de $\mathrm{pH}$ do solo, menor foi a sorção do imazaquin ao solo (F igura 1 e Quadro 2). Considerando o caráter de áci do fraco do imazaquin, houve aumento na percentagem de espécies aniônicas do herbicida $\left(\phi_{a}\right)$ no solo com o aumento do $\mathrm{pH}$ (Quadro 2), resultante da diferença entre o valor de $\mathrm{pK}_{\mathrm{a}}(3,8)$ do grupo carboxílico ácido e o pH do solo (Loux et al., 1989; Regitano et al., 1997). I sso aumentou a repulsão entre as moléculas de imazaquin e os sítios de carga negativa dos colói des do solo. Simultaneamente, a el evação do pH aumentou a polaridade e, com isso, a solubilidade da molécula (Reddy \& Locke, 1996), diminuindo seu caráter hidrofóbico e sua capacidade de sorção. Quando o pH do solo baixou a valores próximos ou abaixo do $\mathrm{pK}_{\mathrm{a}}$ da molécula, a percentagem de espécies aniônicas ( $\phi_{a}$ ) diminuiu (Quadro 2 ), aumentando significativamente a percentagem de espécies neutras em solução e, conseqüentemente, a sorção de imazaquin aos colóides (Ladl ie et al ., 1976; Kells et al., 1980; Nicholls \& Evans, 1991). Nas duas amostras (superfície e profundidade), praticamente não houve diferenças entre os valores dos coeficientes

Quadro 1. Atributos físicos e químicos do Latossolo Vermelho Acriférrico

\begin{tabular}{|c|c|c|c|c|c|c|c|c|c|c|c|}
\hline Camada & PESN & C.O. & Argila & $\mathbf{F e}_{\mathbf{s}}$ & $\mathbf{F e}_{\mathbf{d}}$ & $\mathbf{A l}_{\mathrm{s}}$ & $\mathbf{A} \mathbf{l}_{\mathrm{d}}$ & Gibbsita & Caulinita & $\mathbf{K i}$ & $\mathbf{K r}$ \\
\hline $\mathrm{m}$ & & 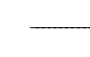 & 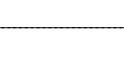 & $\mathrm{g} \mathrm{kg}^{-1}$ & solo - & 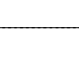 & - & $\mathrm{g} \mathrm{kg}^{-1} \mathrm{~d}$ & $\operatorname{argila}^{(1)}$ & & \\
\hline $0-0,2$ & 3,6 & 20 & 600 & 321 & 131 & 248 & 26 & 375 & 172 & 0,77 & 0,42 \\
\hline $1,0-1,4$ & 5,7 & 5 & 640 & 338 & 158 & 257 & 21 & 431 & 216 & 0,75 & 0,41 \\
\hline
\end{tabular}

(1) argila deferrificada; PESN = ponto de efeito salino nulo; C.O. =carbono orgânico; $\mathrm{Fe}_{\mathrm{s}}=$ óxido de ferro obtido após ataque sulfúrico $\left(\mathrm{Fe}_{2} \mathrm{O}_{3}\right) ; \mathrm{Fe}_{\mathrm{d}}=$ ferro extraído com ditionito-citrato-bicarbonato de sódio; $\mathrm{Al}_{\mathrm{s}}=$ óxido de alumínio obtido após ataque sulfúrico $\left(\mathrm{Al}_{2} \mathrm{O}_{3}\right)$; $\mathrm{Al}_{\mathrm{d}}=$ alumínio extraído com ditionito-citrato-bicarbonato de sódio; $\left.\mathrm{Ki}=\left[\mathrm{SiO}_{2} / \mathrm{Al}_{2} \mathrm{O}_{3}\right) 1,7\right]$ e $\mathrm{Kr}=\left\{\left(\mathrm{SiO}_{2} / 60\right) /\left[\left(\mathrm{Al}_{2} \mathrm{O}_{3} / 102\right)+\left(\mathrm{Fe}_{2} \mathrm{O}_{3} /\right.\right.\right.$ 160)]\}. 
Quadro 2. Parâmetros da sorção e extração de i mazaquin em um Latossolo Vermelho Acriférrico e alguns atributos do solo, considerando diferentes valores de pH do solo

\begin{tabular}{|c|c|c|c|c|c|c|c|c|}
\hline pH & $\Psi_{0}$ & $\phi a$ & $\mathbf{K}_{\mathbf{d}}$ & Koc & $K_{f}$ & $\mathbf{N}$ & $\mathbf{R}^{2}$ & Imazaquin extraído \\
\hline & $\mathrm{mV}$ & $\%$ & $\longrightarrow \mathrm{Lk}$ & - & $\mu \mathrm{mol}^{1-\mathrm{N}} \mathrm{L}^{\mathrm{N}} \mathrm{kg}^{-1}$ & & & $\%$ \\
\hline 3,4 & +9 & 28,47 & $3,23 a^{(1)}$ & 161,5 & 4,65 & 0,86 & 0,999 & $66,34 \pm 3,61$ \\
\hline 4,4 & -49 & 79,92 & $1,27 \mathrm{~b}$ & 63,5 & 1,72 & 0,89 & 0,998 & $81,91 \pm 1,93$ \\
\hline 5,9 & -137 & 99,21 & $0,41 \mathrm{c}$ & 20,5 & 0,53 & 0,91 & 0,991 & $86,62 \pm 2,25$ \\
\hline 7,6 & -238 & 99,98 & $0,20 \mathrm{~d}$ & 10,0 & 0,26 & 0,88 & 0,925 & $88,93 \pm 3,31$ \\
\hline 3,5 & +128 & 33,39 & $4,44 \mathrm{a}^{(1)}$ & 880,0 & 5,91 & 0,87 & 1,000 & $53,14 \pm 4,97$ \\
\hline 5,1 & +30 & 95,23 & $1,83 \mathrm{~b}$ & 366,0 & 2,17 & 0,94 & 0,999 & $80,91 \pm 3,29$ \\
\hline 6,9 & -74 & 99,92 & $0,30 \mathrm{c}$ & 60,0 & 0,31 & 0,97 & 0,955 & $86,48 \pm 2,72$ \\
\hline 7,7 & -119 & 99,99 & $0,20 \mathrm{c}$ & 40,0 & 0,19 & 0,98 & 0,961 & $85,52 \pm 3,00$ \\
\hline
\end{tabular}

(1) Médias com mesma letra não diferem estatisticamente a $5 \%$ pelo teste de Tukey dentro de cada profundidade. $\Psi_{0}=$ potencial elétrico superficial $=59,1 \times(\mathrm{PESN}-\mathrm{pH}) ; \phi_{\mathrm{a}}=$ espécies aniônicas de imazaquin $=\left(1-\phi_{\mathrm{n}}\right) \times 100$, em que $\phi_{\mathrm{n}}=$ espécies neutras de

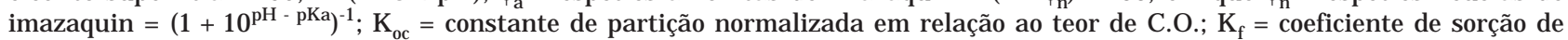
Freundlich; $K_{d}=$ coeficiente de partição (para $\mathrm{N}=1$ ) e $\mathrm{N}=$ declividade da isoterma.

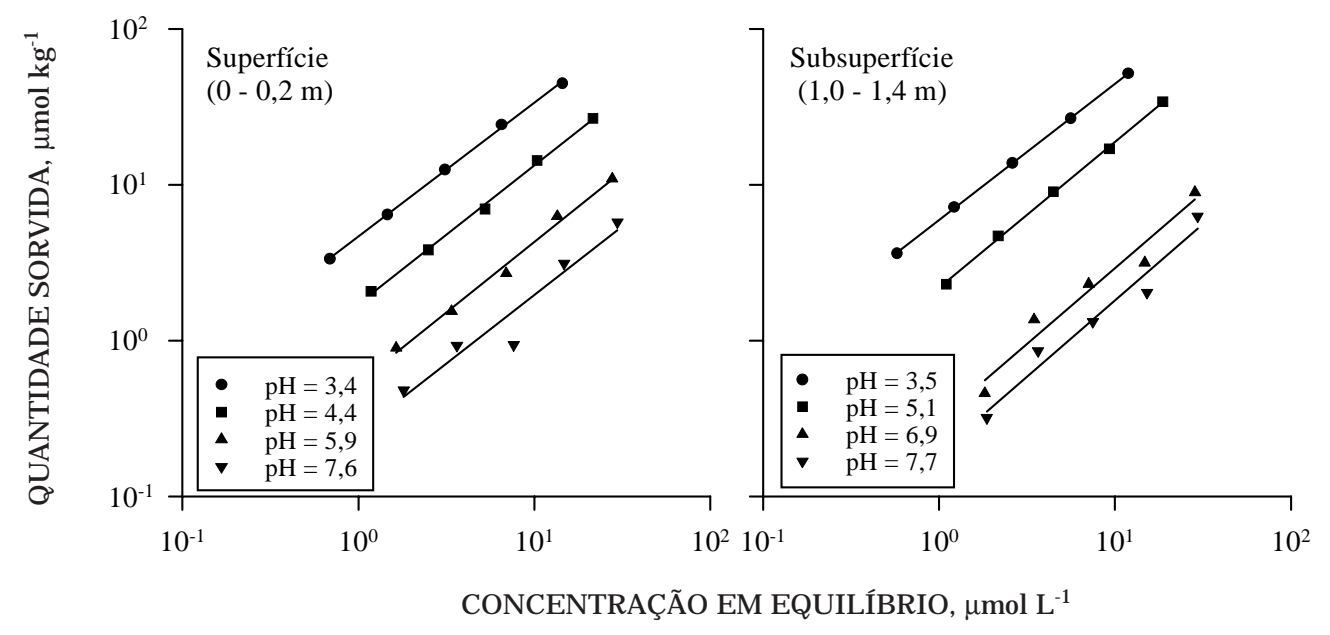

Figura 1. Influência do pH do solo na sorção de imazaquin (ajustada à equação de Freundlich, na forma linear) em amostras de um Latossolo Vermelho Acriférrico.

departição $\left(\mathrm{K}_{\mathrm{d}}\right)$ quandoo $\mathrm{pH}$ do solo foi duas unidades superior aoval or de $\mathrm{pK}_{\mathrm{a}}$ da molécula (istoé, $\mathrm{pH} \geq 5,8$ ) (Quadro 2). Nestes casos, mais de $99 \%$ das moléculas de imazaquin estavam na forma aniônica (Leeet al., 1990; Regitano et al., 1997).

A discussão anterior considerou apenas o efeito do pH dosolo na dissociação (especiação) da molécula de imazaquin, ignorando seu efeito nas cargas variáveis do solo em estudo. No entanto, o valor de $\mathrm{pH}$ do solo também influenciou diretamente o potencial elétrico de solos com cargas variáveis. A não-conformidade das curvas ao model o de Regitano et al. (1997) (Figura 2), que estima a sorção do imazaquin somente pela especiação de sua molécula (aniônica ou neutra) esua partição na fração orgânica do solo, evidenciou que outros atributos do solo estariam influenciando a capacidade sortiva dos colóides em profundidade.

Na camada de pH entre 3,8 e 5,7, a carga líquida positiva do sol ofoi o principal fator responsável pelo aumento da sorção, pois a mai oria das moléculas de imazaquin encontrava-se na forma aniônica, o que favoreceu as atrações el etrostáticas. Para val ores de pH abaixo de 3,8, os colóides do solo continuariam carregados positivamente, mas a maioria das moléculas de imazaquin estaria na forma neutra, minimizando as influências das interações eletrostáticas. Portanto, o contínuo aumento na sorção a val ores baixos de $\mathrm{pH}(\mathrm{pH} \leq 3,8)$ poderia ser explicado pela troca de ligantes, uma vez que a densidade de el étrons da ligação entre $\mathrm{Fe}$ e-O ou Al-O diminuiu eos átomos de oxigênio poderiam ser trocados na forma 
de $\mathrm{OH}^{-}$ou $\mathrm{OH}_{2}$ com o grupo funcional carboxílico da molécula (Evanko \& Dzombak, 1998).

Na faixa de $\mathrm{pH}$ entre 3,5 e 5,8 (Figura 2), a maior quantidade de gibbsita e óxidos de ferro e a menor quantidade de C.O. foram, provavelmente, os responsáveis pela maior sorção em profundidade, sendo os valores ainda superiores aos do modelo.

Como seria de esperar, a percentagem de imazaquin extraída do solo aumentou com o pH, devido à menor força de ligação entre as moléculas do pesticida e os colói des dosolo (Baughman \& Shaw, 1996) (Quadro 2). No valor mais baixo de $\mathrm{pH}$, a percentagem de imazaquin extraída foi menor em subsuperfície, em virtude, provavel mente, da maior quantidade de interações específicas com os óxidos, favor edidas pela menor quantidadedeC.O. (Quadro 1).

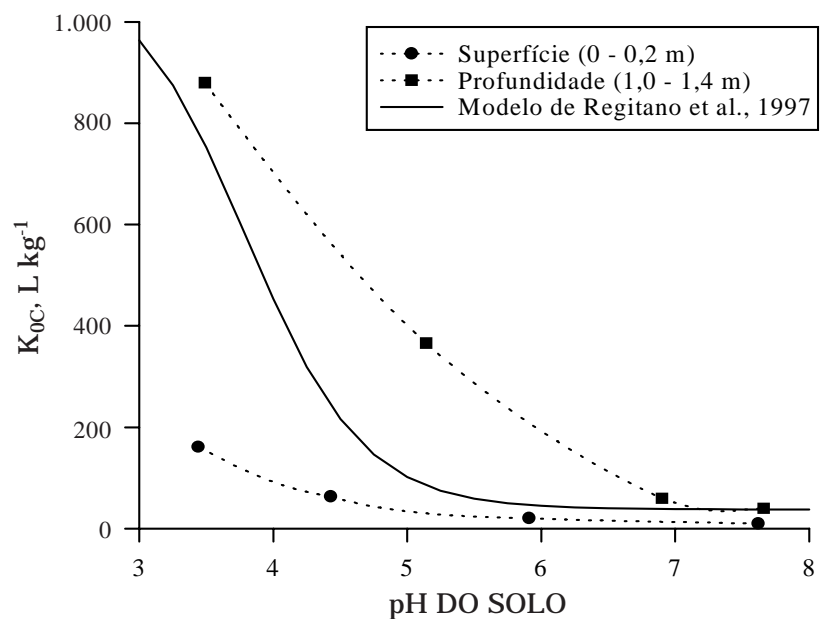

Figura 2. Sorção de imazaquin normalizada em relação ao teor de Carbono Orgânico $\left(\mathrm{K}_{o c}\right)$, considerando o pH de um Latossolo Vermelho Acriférrico.

\section{CONCLUSÕES}

1. O herbicida imazaquin apresentou baixa sorção neste solo de carga variável.

2. À medida que diminuiu o pH do solo, aumentou a sorção do imazaquin.

3. Quando a carga elétrica líquida do solo foi positiva, não foi possível predizer a sorção de imazaquin, considerando apenas a especiação da molécula e sua partição na fração orgânica do solo.

\section{AGRADE CIMENTOS}

À Fundação de Amparo à Pesquisa do Estado de São Paulo(FAPESP), pelofinanciamento da pesquisa (processo no 98/9641-7).

\section{LITE RATURA CITADA}

ALLEONI, L.R.F.\& CAMARGO, O.A. Potencial elétrico superficial e carga elétrica líquida de latossolos ácricos. R. Bras. Ci. Solo, 18:181-185, 1994.

ALLEONI, L.R.F. \& CAMARGO, O.A. Solos ácricos: atributos e manejo. B. Inf. SBCS, 20:9-17, 1995.

BARROW, N.J . A mechanistic model for describing the sorption of phosphate by soils. J. Soil Sci., 34:733-750, 1983.

BARROW, N.J . Modelling the effects of $\mathrm{pH}$ on phosphatesorption by soils. J. Soil Sci., 35:283-297, 1984.

BASHAM, G.; LAVY, T.; OLIVER, L. \& SCOTT, H.D. I mazaquin persistence and mobility in three Arkansas soils. Weed Sci., 35:576-582, 1987.

BAUGHMAN, T.A. \& SHAW, D.R. Effect of wetting/drying cycles on dissipation patterns of bioavailable imazaquin. Weed Sci., 44:380-382, 1996.

BELL, L.C. \& GI LLMAN, G.P. Surfacechargecharacteristics and soil solution composition of highly weathered soils. In: ANDREW, C.S. \& KAMPRATH, E.J ., eds. Mineral nutrition of legumes in tropical and subtropical soils, 1978. Melbourne, CSIRO, 1978. p.37-57.

BEST, J .A. \& WEBER, J.B. Disappearance of s-triazines as affected by soil pH using a balance-sheet approach. Weed Sci., 22:364-373, 1974.

CAMARGO, O.A.; MONIZ, A.C.; J ORGE, J .A. \& VALADARES, J .M.S. Métodos de análise química, mineralógica e física de sol os do Instituto Agronômico de Campinas. Campinas, Instituto Agronômico de Campinas, 1986. 94p. (Boletim Técnico, 106)

COLBERT, F.O.; VOLK, V.V. \& APPLEBY, A.P. Sorption of atrazine, terbutrin, and GS-14254 on natural and limeamended soils. Weed Sci., 23:390-394, 1975.

EMPRESA BRASILEIRA DE PESQUISA AGROPECUÁRIA EMBRAPA. Sistema brasileiro de classificação de solos. Brasília, Produção de I nformação, 1999. 412p.

EVANKO, C.R. \& DZOMBAK, D.A. Influence of structural features on sorption of NOM-analogue organic acids to goethite. Environ. Sci. Technol., 32:2846-2855, 1998.

GOETZ, A.J .; WEHTJ E, G.; WALKER, R.H. \& HAJ EK, B. Soil solution and mobility characterization of imazaquin. Weed Sci., 34:788-793, 1986.

HANG, S.B.; FERREIRO, E.A. \& DE-BUSSETTI, S.G. Movilidad y adsorcion-desorcion de picloram, dicamba e imazaquin. Inv. Agr. Produc. Protec. Veg., 11:345-361, 1996.

KELLS, J J .; RIECK, C.E.; BLEVINS, R.L. \& MUIR, W.M. Atrazine dissipation as affected by surface $\mathrm{pH}$ and tillage. Weed Sci., 28:101-104, 1980.

KOSKINEN, W.C. \& HARPER, S.S. The retention process: mechanisms. In: CHENG, H.H., ed. Pesticides in the soil environment: process, impacts, and modeling. 2.ed. Madison, Soil Science Society of America, 1990. p.51-77.

LADLIE, J .; MEGGITT, W.F.\& PENNER, D. Effect of soil pH on microbial degradation, adsorption, and mobility of metribuzin. Weed Sci., 24:477-481, 1976. 
LEE, L.S.; RAO, P.S.C.; NKEDI-KIZZA, P. \& DELFINO, J .J . Influence of solvent and sorbent characteristics on distribution of pentachlorophenol in octanol-water and soilwater systems. Environ. Sci. Technol., 24:654-661, 1990.

LOUX, M.M.; LIEBL, R.A. \& SLIFE, F.W. Adsorption of imazaquin and imazethapyr on soils, sediments, and selected adsorbents. Weed Sci., 37:712-718, 1989.

NICHOLLS, P.H. \& EVANS, A.A. Sorption of ionisable organic compounds by field soils. Part 1: Acids. Pest. Sci., 33:319330, 1991.

PIRES, N.M.; SILVA, J .F.; SILVA, J .B.; FERREIRA, L.R. \& CARDOSO, A.A. Adsorção e lixiviação de Trifluralin e I mazaquin em diferentes solos. R. Ceres, 44:300-314, 1997.

REDDY, K.N. \& LOCKE, M.A. I mazaquin spray retention, foliar washoff and runoff losses under simulated rainfall. Pest. Sci., 48:179-187, 1996.

REGITANO, J B.; BISCHOFF, M.; LEE, L.S.; REICHERT, J .M. \& TURCO, R.F. Retention of Imazaquin in soil. Environ. Toxicol. Chem., 16:397-404, 1997.
RENNER, K.A.; MEGGITT, W.F. \& PENNER, D. Effect of soil $\mathrm{pH}$ on I mazaquin and I mazethapyr adsorption to soil and phytotoxicity to corn (Zea mays). Weed Sci., 36:78-83, 1988.

RESENDE, M. \& SANTANA, D.P. Uso das relações Ki e Kr na estimativa da mineralogia para classificação dos $L$ atossolos. In: REUNIÃO DE CLASSIFICAÇÃO, CORRELAÇÃO DE SOLOS E INTERPRETAÇÃO DE APTIDÃO AGRÍCOLA, 3., Rio de J aneiro, 1988. Anais. Rio de J aneiro, Empresa Brasileira de Pesquisa Agropecuária, 1988. p.225-232. (Documentos, EMBRAPA-SNLCS, 12)

STOUGAARD, R.N.; SHEA, P.J . \& MARTIN, A.R. Effect of soil type and $\mathrm{pH}$ on adsorption, mobility and efficacy of imazaquin and imazethapyr. Weed Sci., 38:67-73, 1990.

UEHARA, G. Acric properties and their significance to soil classification. In: INTERNATIONAL SOIL CLASSIFICATION WORKSHOP, 8., Rio deJ aneiro, 1986. Proceedings. Rio deJ aneiro, EMBRAPA/SNLCS, 1988. p.1922.

YU, T.R Chemistry of variable charge soils. New York, Oxford University Press, 1997. 505p. 
W. S. D. ROCHA et al. 